\title{
Myosin Light Chain Kinase Knockout Improves Gut Barrier Function and Confers a Survival Advantage in Polymicrobial Sepsis
}

\author{
C Adam Lorentz, ${ }^{1^{*}}$ Zhe Liang,, ${ }^{2 *}$ Mei Meng, ${ }^{3}$ Ching-Wen Chen, ${ }^{2}$ Benyam P Yoseph, ${ }^{2}$ Elise R Breed, ${ }^{2}$ \\ Rohit Mittal, ${ }^{2}$ Nathan J Klingensmith, ${ }^{2}$ Alton B Farris, ${ }^{4}$ Eileen M Burd, ${ }^{4}$ Michael Koval, ${ }^{5}$ Mandy L Ford, ${ }^{6}$ \\ and Craig $M$ Coopersmith ${ }^{2}$
}

${ }^{1}$ Department of Urology; ${ }^{2}$ Department of Surgery and Emory Critical Care Center, Emory University School of Medicine, Atlanta, Georgia, United States of America; ${ }^{3}$ Department of Critical Care Medicine, Shandong Provincial Hospital Affiliated Shandong University, Jinan, China; ${ }^{4}$ Department of Pathology and Laboratory Medicine; ${ }^{5}$ Department of Internal Medicine and Emory Alcohol and Lung Biology Center; and ${ }^{6}$ Department of Surgery and Emory Transplant Center, Emory University School of Medicine, Atlanta, Georgia, United States of America

\begin{abstract}
Sepsis-induced intestinal hyperpermeability is mediated by disruption of the epithelial tight junction, which is closely associated with the perijunctional actin-myosin ring. Myosin light chain kinase (MLCK) phosphorylates the myosin regulatory light chain, resulting in increased permeability. The purpose of this study was to determine whether genetic deletion of MLCK would alter gut barrier function and survival from sepsis. $\mathrm{MLCK}^{-1-}$ and wild-type (WT) mice were subjected to cecal ligation and puncture and assayed for both survival and mechanistic studies. Survival was significantly increased in $\mathrm{MLCK}^{-1-}$ mice ( $95 \%$ versus $24 \%, p<0.0001$ ). Intestinal permeability increased in septic WT mice compared with unmanipulated mice. In contrast, permeability in septic $\mathrm{MLCK}^{-/-}$mice was similar to that seen in unmanipulated animals. Improved gut barrier function in $\mathrm{MLCK}^{-1-}$ mice was associated with increases in the tight junction mediators ZO- 1 and claudin 15 without alterations in claudin 1, 2, 3, 4, 5, 7, 8 and 13, occludin or JAM-A. Other components of intestinal integrity (apoptosis, proliferation and villus length) were unaffected by MLCK deletion, as were local peritoneal inflammation and distant lung injury. Systemic IL-10 was decreased greater than 10-fold in MLCK ${ }^{-1-}$ mice; $^{-}$however, survival was similar between septic $\mathrm{MLCK}^{-1-}$ mice given exogenous IL-10 or vehicle. These data demonstrate that deletion of MLCK improves survival following sepsis, associated with normalization of intestinal permeability and selected tight junction proteins.
\end{abstract}

Online address: http://www.molmed.org

doi: $10.2119 /$ molmed.2016.00256

\section{INTRODUCTION}

Sepsis is life-threatening organ dysfunction secondary to a dysregulated host response to infection (1). Between 230,000 and 370,000 patients die of sepsis annually, and mortality from septic shock is $>40 \%(2,3)$. Aside from antibiotics and fluid resuscitation, management of sepsis currently consists of supportive care, and there are no targeted therapies approved for the management of sepsis (4).

The gut has long been hypothesized to be the "motor" of multiple organ dysfunction (5-11). Gut epithelial integrity is altered in sepsis, with increased apoptosis, decreased villus length and

*These authors contributed equally to this work.

Address correspondence to Craig M Coopersmith, 101 Woodruff Circle, Suite WMB 5105, Atlanta, GA 30322, USA. Phone: (404) 727-4273; Fax: (404) 727-3660;

E-mail: cmcoop3@emory.edu

Submitted December 15, 2016; Accepted for Publication May 31, 2017;

Published Online (www.molmed.org) June 7, 2017.

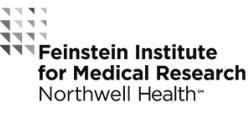

Feinstein Institute
for Medical Research Northwell Health

decreased crypt proliferation (12-17). Notably, sepsis induces intestinal hyperpermeability (18-21). While early studies in critical illness hypothesized that gut barrier damage induces sepsis by translocation of intact bacteria, the reality has turned out to be considerably more complex $(7,22-24)$. The intestine acts as a selective barrier, preventing movement of potentially damaging microbes, toxins and antigens from the gut lumen, while simultaneously allowing paracellular movement of water, solutes and immune-modulating factors (25-27). Luminal contents can exit the internal environment of the gut into the local environment or the systemic circulation via the portal circulation or mesenteric lymph, where they can cause distant organ damage or alter inflammation. 
Notably, interventions aimed at restoring the intestinal barrier have been associated with improvements in survival in animal models of critical illness $(28,29)$.

Paracellular permeability is regulated via a number of complementary mechanisms (30,31), including tight junction proteins and tight junction-associated proteins, which regulate molecular flux based upon both size and charge (32-34). The tight junction complex is intimately associated with the perijunctional actin-myosin ring. Myosin light chain kinase (MLCK) phosphorylates the myosin regulatory light chain, resulting in contraction of the actin-myosin ring, subsequently increasing permeability at the cell-cell junction (35). MLCK activation is commonly seen with bacterial infection, leading to alterations in tight junctions $(36,37)$. MLCK activation is also associated with increases in IL-6, TNF and IL-1 $\beta$ that further activate MLCK. This, in turn, increases permeability, in part via altered ZO-1 protein dynamics and occludin removal (38-40), and ultimately leads to an amplification of the systemic inflammatory response via a feed-forward mechanism. Inhibition of MLCK can reverse these changes to the intestinal tight junction $(41,42)$. The aim of this study was to determine whether genetic deletion of MLCK reverses sepsis-induced intestinal hyperpermeability and whether this would confer a survival advantage in this highly lethal disease.

\section{MATERIALS AND METHODS}

\section{Animals}

Six- to 12-wk-old male and female mice with a genetic deletion of the long isoform of MLCK on a C57Bl/ 6 background $\left(\mathrm{MLCK}^{-/-}\right.$, a generous gift from Dr Martin Watterson, Northwestern University [43]) and wild-type (WT) mice on the same genetic background were used in all experiments. Mice were bred to genetically identical animals (i.e., $\mathrm{MLCK}^{-/-}$to $\mathrm{MLCK}^{-/-}$and WT to WT) throughout the course of the experiments. Mice were kept on a strict day/ night light cycle and had free access to food and water throughout the course of the experiments. All experiments were performed in accordance with the National Institutes of Health Guidelines for the Use of Laboratory Animals and were approved by the Institutional Animal Care and Use Committee at Emory University School of Medicine (protocol DAR-2002717-042217GN).

\section{Sepsis Model}

Animals were subjected to cecal ligation and puncture (CLP), a model of fecal peritonitis-induced sepsis (44). Under isoflurane anesthesia, a midline incision was performed and the cecum was externalized. The cecum was ligated distal to the ileocecal valve using a 4-0 silk suture and punctured twice distal to the ligation point with a 25-gauge needle. After a small amount of stool was extruded, the cecum was replaced into the abdomen in its normal position, and the abdomen was closed in layers. Immediately postoperatively, animals received $1 \mathrm{~mL}$ of sterile saline subcutaneously for fluid resuscitation and were allowed to recover on a warming pad. All animals were given ceftriaxone $25 \mathrm{mg} / \mathrm{kg}$ (Sigma-Aldrich, St. Louis, MO) and metronidazole $12.5 \mathrm{mg} / \mathrm{kg}$ (Apotex Corp, Weston, FL) postoperatively and every $12 \mathrm{~h}$ for $2 \mathrm{~d}$ to mimic management of the human disease, where fluid resuscitation and antibiotics are the mainstay of early sepsis treatment (45). To minimize animal suffering, all mice received buprenex immediately postoperatively $(0.1 \mathrm{mg} / \mathrm{kg}$; McKesson Medical, San Francisco, CA). Unless explicitly stated otherwise, animals were euthanized $24 \mathrm{~h}$ after CLP.

\section{Intestinal Permeability}

Mice were given $0.5 \mathrm{~mL}$ of fluorescein isothiocyanate-dextran (FD-4) $(22 \mathrm{mg} / \mathrm{mL}$; Sigma-Aldrich) via oral gavage $5 \mathrm{~h}$ prior to being euthanized. Serum collected after that was diluted with an equivalent volume of phosphate-buffered saline (PBS). The concentration of FD-4 in the serum was determined using fluorospectrometry at excitation/emission wavelengths of $470 / 515 \mathrm{~nm}$ with standard curve serial dilutions (BioTex Synergy HT, Winooski, VT).

\section{Western Blot Analysis}

Jejunal tissue was harvested at the time of euthanasia and immediately flash-frozen in liquid nitrogen. Samples were later thawed and immersed in lysis buffer (50 mM Tris-HCl, pH 7.4; 150 mM Triton X-100; 50mM dithiothreitol 50 $\mu \mathrm{g} / \mathrm{mL}$ leupeptin; $5 \mathrm{mM}$ phenylmethylsulfonyl fluoride; $1 \mathrm{mM} \mathrm{NaF}$; $1 \mathrm{mM} \mathrm{Na}_{3} \mathrm{VO}_{4}$ ) in an ice bath and homogenized using a sonicator. Samples were then centrifuged at $4^{\circ} \mathrm{C}$ for $5 \mathrm{~min}$, and the supernatant was analyzed for protein concentration using fluorometry. Samples were then combined with Laemmli buffer at a 1:2 ratio and heated at $95^{\circ} \mathrm{C}$ for $5 \mathrm{~min}$, after which they were loaded onto polyacrylamide gels (Bio-Rad, Hercules, CA) and run at $180 \mathrm{~V}$ for $45 \mathrm{~min}$. Samples were then transferred to Immuno-Blot polyvinyledene difluoride membranes (Bio-Rad) at $80 \mathrm{~V}$ for $2 \mathrm{~h}$. Membranes were blocked using 5\% nonfat milk at room temperature for $1 \mathrm{~h}$ and then probed with the following primary anti-rabbit antibodies at $4^{\circ} \mathrm{C}$ overnight: anti-claudin 1 , anticlaudin 3, anti-claudin 4, anti-claudin 5, anti-claudin 7, anti-claudin 8, anticlaudin 13 , anti-claudin 15 , anti-occludin, anti-ZO-1, anti- $\beta$-actin (Cell Signaling Technology, Danvers MA), anti-claudin 2 and anti-JAM-A (Abcam, Cambridge, MA). Membranes were then washed with tris-buffered saline with $0.1 \%$ Tween 20 (Sigma) and incubated with horseradish peroxidase (HRP)-conjugated goat anti-rabbit immunoglobulin for $1 \mathrm{~h}$. Protein bands were visualized using a chemiluminescent developing system (Thermo Fisher Scientific, Waltham MA) and exposed to x-ray film. Resulting images were scanned into a computer and analyzed using densitometric software (ImageJ, National Institutes of Health, Bethesda, MD). Intensity of the protein bands of interest was internally normalized to a reference housekeeping protein ( $\beta$-actin). All groups were transformed by dividing each group by the mean of the control group. 
Villus length. Villus length was measured as the distance in $\mu \mathrm{m}$ from the crypt neck to the villus tip in 12-16 well-oriented contiguous crypt-villus units using Nikon Elements imaging software (Nikon Instruments, Melville, NY).

Intestinal apoptosis. Apoptotic cells were quantified in the jejunum using two complementary techniques: active caspase 3 staining and morphologic analysis of hematoxylin and eosin (H\&E)stained sections (46). For the former, sections were deparaffinized and rehydrated. Following a $10 \mathrm{~min}$ incubation in 3\% hydrogen peroxide, sections were placed in Antigen Decloaker (Biocare Medical, Concord, CA) and heated in a pressure cooker for $45 \mathrm{~min}$. Sections were blocked with $20 \%$ normal goat serum (Vector Laboratories, Burlingame, CA) and incubated overnight with rabbit polyclonal anti-active caspase 3 (1:100; Cell Signaling, Beverly, MA) at $4^{\circ} \mathrm{C}$. Sections were then incubated with goat anti-rabbit biotinylated secondary antibody (1:200; Accurate Chemical and Scientific, Westbury, NJ) for $60 \mathrm{~min}$ followed by HRP-labeled streptavidin (Accurate Chemical and Scientific) for $60 \mathrm{~min}$. Sections were developed with diaminobenzidine and counterstained with hematoxylin. Apoptotic cells were identified on H\&E-stained sections via characteristic morphological changes (17). Apoptotic cells were quantified in 100 well-oriented contiguous crypt-villus units for both techniques by an investigator blinded to sample identity.

Intestinal proliferation. To label cells in $S$ phase, mice received an intraperitoneal injection of 5-Bromo-2'deoxyuridine (BrdU, $5 \mathrm{mg} / \mathrm{mL}$ diluted in normal saline; Sigma) $90 \mathrm{~min}$ prior to being euthanized (47). After sections were deparaffinized and rehydrated, they were incubated in $1 \%$ hydrogen peroxide for $15 \mathrm{~min}$, then immersed in Antigen Decloaker and heated in a pressure cooker for $45 \mathrm{~min}$. Sections were then placed in Protein Block (Dako, Carpinteria, CA) for $10 \mathrm{~min}$, and incubated with rat monoclonal anti-BrdU overnight at $4^{\circ} \mathrm{C}$ (1:500; Accurate Chemical and
Scientific). Sections were then incubated with goat anti-rat biotinylated secondary antibody (1:500) for $60 \mathrm{~min}$, followed by HRP-labeled streptavidin for $60 \mathrm{~min}$. Sections were developed with diaminobenzidine and counterstained with hematoxylin, and BrdU-stained cells were quantified in 100 well-oriented contiguous crypts.

Tight junction immunohistochemistry. Sections were deparaffinized, rehydrated, washed with PBS and then incubated with $20 \%$ goat serum albumin. Sections were stained with rabbit anti-ZO-1 or anti-claudin 15 (1:1000; Cell Signaling Technology) overnight at $4^{\circ} \mathrm{C}$. After washing with PBS, samples were incubated with Alexa Fluor ${ }^{\circledR} 488$ donkey anti-rabbit antibody (1:500, Biolegend, San Diego, CA) for $1 \mathrm{~h}$ at room temperature. Samples were then washed again in PBS and counterstained with 4',6-diamidino-2-phenylindole (Fisher Scientific, Pittsburgh, PA).

Bacterial cultures. Serum and peritoneal fluid samples were obtained for bacterial culture. Peritoneal fluid was obtained by lavaging the abdomen with $1 \mathrm{~mL}$ of sterile saline. All samples for culture were serially diluted in sterile saline and plated on sheep's blood agar plates (Remel, Lenexa, KS). Plates were incubated overnight at $37^{\circ} \mathrm{C}$ in $5 \% \mathrm{CO}_{2}$ and colony counts were determined.

Lung wet-to-dry ratios, myeloperoxidase activity, and histology. The left lung from each mouse was harvested and weighed to determine a wet weight. The lung was then dried in an oven at $115^{\circ} \mathrm{C}$ for $24 \mathrm{~h}$ and reweighed to establish a dry weight, after which a ratio of wet weight to dry weight was calculated.

The right lung was homogenized in $50 \mathrm{mM}$ sodium phosphate buffer containing $0.5 \%$ hexadecyltrimethylammonium bromide, heated for $2 \mathrm{~h}$ at $55^{\circ} \mathrm{C}$, centrifuged at 13,000 rpm for $20 \mathrm{~min}$ at $4^{\circ} \mathrm{C}$, and then supernatants were collected.

Bronchoalveolar lavage (BAL) fluid was obtained by cannulating the trachea and lavaging the lungs with $1 \mathrm{~mL}$ of sterile saline. Samples were centrifuged at $5,000 \mathrm{rpm}$ for $5 \mathrm{~min}$. Following addition of substrate buffer containing O-dianisidine and $0.0005 \%$ hydrogen peroxide, myeloperoxidase (MPO) activity was measured at $460 \mathrm{~nm}$ wavelength over 6 min (Bio-Tek Instruments $\mu$ Quant Microplate Spectrophotometer, Winooski, VT). MPO activity was calculated as optical density/minute/mg of lung tissue or per $\mu \mathrm{L}$ of BAL fluid.

Lung histology was also examined by a pathologist $(\mathrm{ABF})$ blinded to sample identity. Lung histology was scored based upon proportion of tissue with inflammation as follows: no inflammation (0), 1-4\% (1), 5-10\% (2), 11-20\% (3), $21-100 \%$ (4).

Cytokines. Blood, peritoneal fluid and BAL fluid were collected at the time of euthanasia and then centrifuged at 5,000 rpm for $5 \mathrm{~min}$. Concentrations of TNF, IL-6, IL-10, IL-13 and IFN- $\gamma$ were measured using a multiplex cytokine assay (Bio-Rad, Hercules, CA) according to the manufacturer's instructions. All samples were run in duplicate.

Leukocyte analysis. Splenocytes were isolated, placed on ice for $30 \mathrm{~min}$ and stained for anti-CD3 (BD Pharmingen, San Diego, CA), anti-CD4 (BD Pharmingen), anti-CD8 (Invitrogen ${ }^{\mathrm{TM}}$, ThermoFisher Scientific, Waltham, MA), anti-CD19 (BioLegend, San Diego, CA), anti-CD11c (eBioscience, Santa Clara, CA), anti-CD25 (BD Pharmingen) and anti-CD69 (BioLegend). Absolute numbers were calculated using CountBright $^{\mathrm{TM}}$ Absolute Counting Beads (Life Technologies, Waltham, MA) according to the manufacturer's instructions. Samples were analyzed on an LSRII flow cytometer (BD Biosciences). Data were analyzed using FlowJo software (Tree Star, San Carlos, CA).

Exogenous IL-10. $\mathrm{MLCK}^{-/-}$mice were given either exogenous IL10 (20 $\mu \mathrm{g}$; BioLegend) or vehicle via intraperitoneal injection immediately after the onset of sepsis utilizing a published protocol (48). Mice were treated with fluids, antibiotics and pain medicine and followed $7 \mathrm{~d}$ for survival.

IL-10 neutralization. Mice were given anti-IL-10 monoclonal antibody $(250 \mu \mathrm{g}$, 
Genzyme, Cambridge, MA) or isotype control via intraperitoneal injection $12 \mathrm{~h}$ following CLP. Mice were treated with identical fluids, antibiotics and pain medicine as in the above experiments and followed $7 \mathrm{~d}$ for survival.

\section{Statistics}

Data were analyzed using the statistical software program Prism 6.0 (GraphPad, San Diego, CA) and are presented as mean \pm standard error of the mean. Data sets were tested for Gaussian distribution using the D'Agostino-Pearson omnibus normality test. Comparison between groups with a Gaussian distribution was performed using the Student $t$ test. Comparison between groups that did not have a Gaussian distribution was performed using the Mann-Whitney test. Survival was analyzed using the logrank test. A $p$ value $<0.05$ was considered to be statistically significant.

All supplementary materials are available online at www.molmed.org.

\section{RESULTS}

\section{Survival Is Improved in $\mathrm{MLCK}^{-/-}$Mice Following Sepsis}

Mortality was significantly decreased in $\mathrm{MLCK}^{-/-}$mice compared with WT mice following CLP (Figure 1). In all, $95 \%$ of $\mathrm{MLCK}^{-/-}$mice survived for $7 \mathrm{~d}$ after their septic insult compared with only $24 \%$ of WT mice.

\section{Intestinal Permeability Is Normalized in $\mathrm{MLCK}^{-/-}$Mice Following Sepsis, Associated with Increases in ZO-1 and Claudin 15}

Baseline permeability was similar in unmanipulated $\mathrm{MLCK}^{-/-}$mice and WT mice (Figure 2). Compared to unmanipulated animals, in WT mice permeability was significantly increased following CLP at 6, 12 and $24 \mathrm{~h}$ and returned to baseline by $48 \mathrm{~h}$. In contrast, permeability was significantly lower in septic $\mathrm{MLCK}^{-/-}$mice than in WT mice at 6, 12 and $24 \mathrm{~h}$ (Figure 2). Notably, permeability was not altered by CLP in $\mathrm{MLCK}^{-/-}$ mice, as the concentration of FD-4 in the blood at all time points following gavage was similar in both unmanipulated and septic animals.

To determine the potential mechanisms by which permeability was normalized in $\mathrm{MLCK}^{-/-}$mice, an analysis of jejunal tight junctions was undertaken $24 \mathrm{~h}$ after CLP. ZO-1 and claudin 15 levels were increased in septic $\mathrm{MLCK}^{-/-}$ mice compared with WT mice by both Western blot and immunohistochemistry, although no change in subcellular localization was identified (Figure 3). In contrast, no differences were seen in protein levels of claudin 1, 2, 3, 4, 5, 7, 8 and 13, occludin and JAM-A (Figure 4).

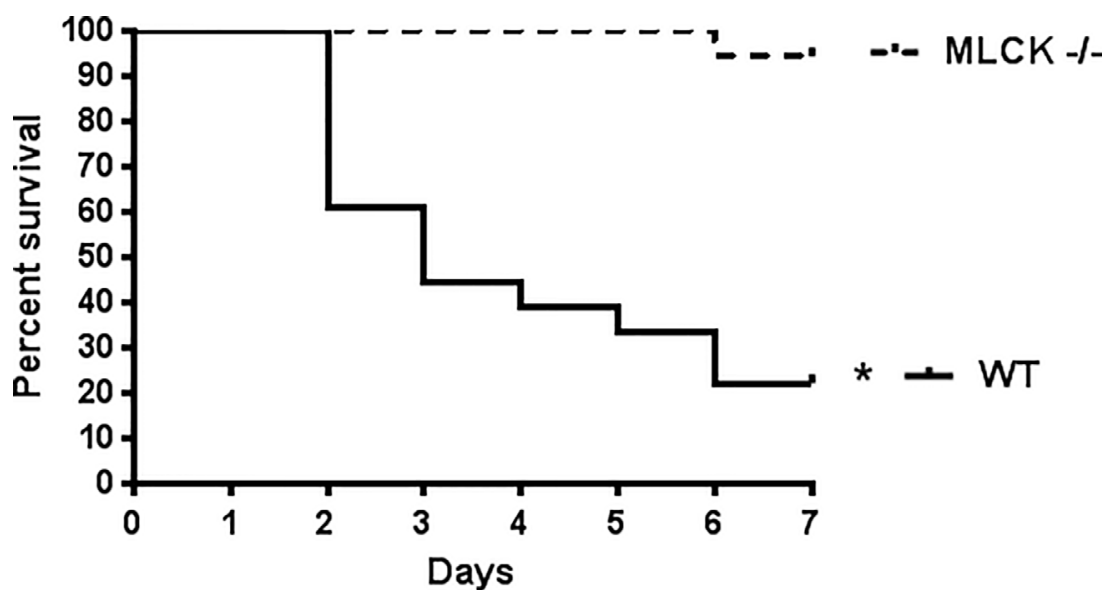

Figure 1. Effect of genetic deletion of MLCK on survival. Seven-day survival was markedly improved in $\mathrm{MLCK}^{-1-}$ mice following CLP compared with WT mice $(n=18 /$ group, $p<0.0001)$.
Intestinal Apoptosis, Proliferation and Villus Length Are Unaffected in $\mathrm{MLCK}^{-/-}$Mice Following Sepsis

To determine whether genetic deletion of MLCK altered components of gut integrity beyond permeability, $\mathrm{MLCK}^{-/}$ mice and WT mice were subjected to CLP and euthanized $24 \mathrm{~h}$ later. No differences were noted in gut epithelial apoptosis (Figures 5A, B), proliferation (Figure 5C) or villus length (Figure 5D) between $\mathrm{MLCK}^{-/-}$mice and WT mice $24 \mathrm{~h}$ after CLP.

\section{Lung Wet-to-dry Ratio, Neutrophil Infiltration, BAL Cytokines and Histology Are Unaffected in $\mathrm{MLCK}^{-/}$ Mice Following Sepsis}

To determine whether genetic deletion of MLCK altered lung injury, $\mathrm{MLCK}^{-/-}$ mice and WT mice were subjected to CLP and euthanized $24 \mathrm{~h}$ later. No differences were noted in lung wet-to-dry ratio (Figure 6A), myeloperoxidase assay in either lung (Figure 6B) or BAL fluid (Figure 6C), or in BAL cytokines TNF, IL-6, IL-10, IL-13 and IFN- $\gamma$ (Figures $6 \mathrm{D}-\mathrm{H}) 24 \mathrm{~h}$ after CLP. Minimal lung inflammation was seen in $\mathrm{MLCK}^{-/-}$mice and WT mice (average lung inflammation score $0.8 \pm 0.2$ versus $1.4 \pm 0.5$, $p=0.51$ ).

\section{Peritoneal Fluid Cultures and Cytokines Are Unaffected in $\mathrm{MLCK}^{-/-}$ Mice Following Sepsis Except for a Decrease in IL-6}

To determine whether genetic deletion of MLCK altered local bacterial clearance and inflammatory response, $\mathrm{MLCK}^{-/-}$ mice and WT mice were subjected to CLP and had their peritoneal fluid sampled $24 \mathrm{~h}$ later. Bacterial burden was similar between $\mathrm{MLCK}^{-/-}$mice and WT mice $24 \mathrm{~h}$ after CLP (Figure 7A). Levels of IL-6 were lower in the peritoneal fluid of septic $\mathrm{MLCK}^{-/-}$mice compared with septic WT mice (Figure 7B), although peritoneal IL-6 levels were similar in unmanipulated $\mathrm{MLCK}^{-/-}$and WT mice (data not shown). No differences were noted in TNF, IL-10, IL-13 or IFN- $\gamma$ in septic $\mathrm{MLCK}^{-/-}$and WT mice (Figures $7 \mathrm{C}-\mathrm{F}$ ). 


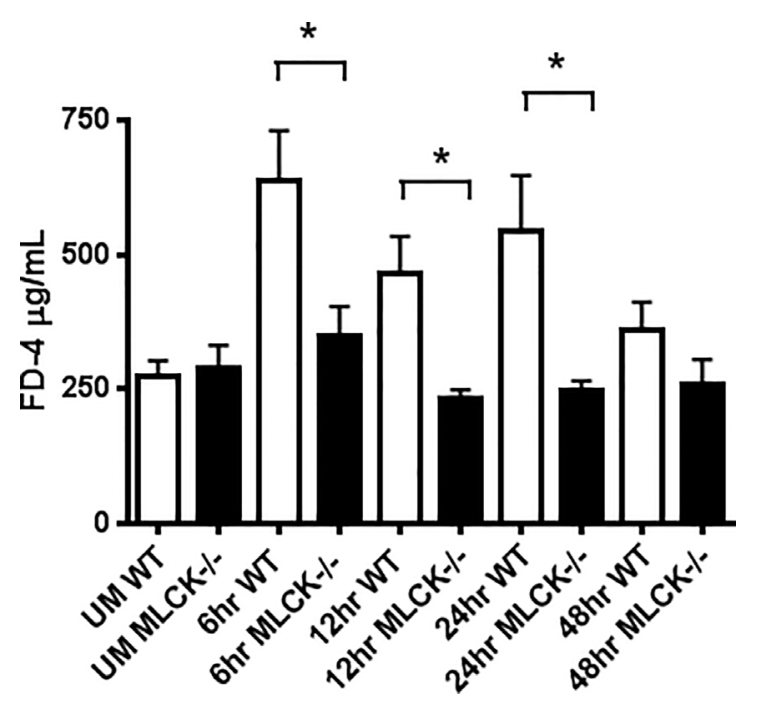

Figure 2. Effect of genetic deletion of MLCK on intestinal permeability 6, 12, 24 and $48 \mathrm{~h}$ after CLP. Basal permeability to FD-4 was similar in unmanipulated (UM) $\mathrm{MLCK}^{-1-}$ and WT mice ( $n=5-6$ /group, $p=0.2$ ). Sepsis worsened intestinal permeability 6,12 and $24 \mathrm{~h}$ after sepsis, which returned to basal levels $48 \mathrm{~h}$ after CLP. In contrast, permeability remained at basal levels in $\mathrm{MLCK}^{-1-}$ mice following CLP. Intestinal permeability was statistically lower in $\mathrm{MLCK}^{-1-}$ mice than WT mice at all time points where sepsis induced intestinal hyperpermeability ( $n=7-9 /$ group at $6 \mathrm{~h}, \mathrm{n}=14-16 /$ group at $12 \mathrm{~h}, \mathrm{n}=11-14$ /group at $24 \mathrm{~h}, \mathrm{n}=7-10$ / group at $48 \mathrm{~h} ; \mathrm{p}=0.02, p=0.0009, p=0.02, p=0.18$ when comparing $\mathrm{MLCK}^{-/-}$and WT mice at $6,12,24$ and $48 \mathrm{~h}$ ).

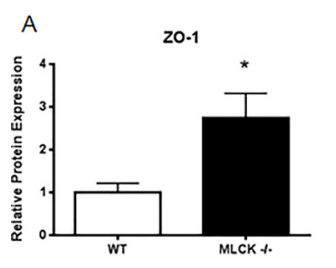

$$
\text { B }
$$
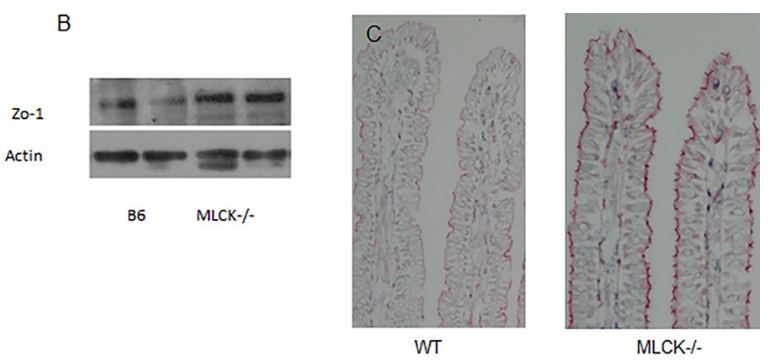

$D$

$E$
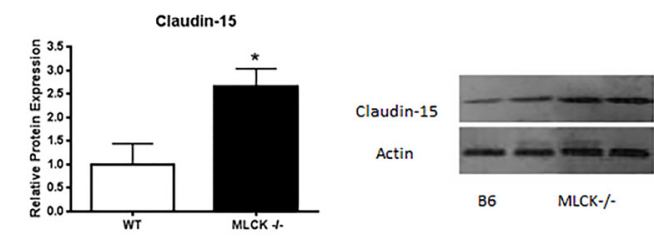

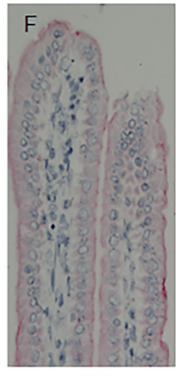

WT

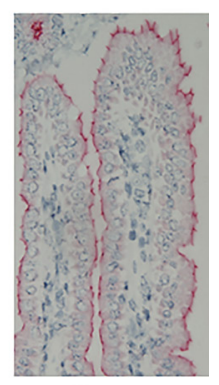

MLCK-1-

Figure 3. Effect of genetic deletion of MLCK on ZO-1 and claudin $1524 \mathrm{~h}$ after CLP. Protein levels of (A) ZO-1 and (B) claudin 15 in jejunal tissue were higher in $\mathrm{MLCK}^{-1-}$ mice than WT mice following CLP ( $n=3-6 /$ group, $p=0.03$ and 0.048 , respectively). (B,E) Representative Western blots are shown for both of these proteins with actin controls. Although immunohistochemical staining was more pronounced for (C) ZO-1 and claudin (F) 15 on the villi of $\mathrm{MLCK}^{-1-}$ mice, no apparent difference in subcellular localization was detected.

Systemic IL-10 Levels Are Decreased in $\mathrm{MLCK}^{-/-}$Mice but Survival Is Unchanged by Giving Exogenous IL-10

To determine whether genetic deletion of MLCK altered systemic bacterial clearance and inflammatory response, $\mathrm{MLCK}^{-1-}$ mice and WT mice were subjected to CLP and had their blood sampled $24 \mathrm{~h}$ later. Blood cultures showed similar growth between $\mathrm{MLCK}^{-/-}$mice and WT mice $24 \mathrm{~h}$ after CLP (Figure 8A). Levels of serum IL-10 were decreased greater than 10-fold in $\mathrm{MLCK}^{-1-}$ mice compared with WT mice (Figure 8B), although levels of IL-10 were similar in unmanipulated $\mathrm{MLCK}^{-/-}$and WT mice (data not shown). Giving exogenous IL-10 to septic $\mathrm{MLCK}^{-/-}$mice did not change $7 \mathrm{~d}$ survival compared with septic $\mathrm{MLCK}^{-/-}$mice given vehicle (Figure 8C). In addition, giving IL-10 neutralizing antibody to both $\mathrm{MLCK}^{-1-}$ mice and WT mice completely eliminated the survival benefit conferred by genetic deletion of MLCK, resulting in a nonsignificant trend toward worsened survival in $\mathrm{MLCK}^{-1-}$ mice (Figure 8D). Levels of IL-6 in the serum were also lower in septic $\mathrm{MLCK}^{-1-}$ mice compared with WT mice (Figure 8E), but no differences were noted in TNF, IL-13 or IFN- $\gamma$ (Figures 8 F-H).

\section{CD4 + T cell Counts Are Higher and Percentages of CD4 + CD25 + and CD8 + CD25 + T Cells Are Lower in $\mathrm{MLCK}^{-/-}$Mice Following Sepsis}

$\mathrm{CD} 4+\mathrm{T}$ cell counts were higher in septic $\mathrm{MLCK}^{-/-}$mice $24 \mathrm{~h}$ after CLP (Figure 9A), without differences in $\mathrm{CD} 8+, \mathrm{CD} 11 \mathrm{C}+$ or CD19 + counts (Figure 9B-D). Percentages of both CD4 + CD25 + and CD8 + CD25 + were lower in septic $\mathrm{MLCK}^{-1-}$ mice (Figure 9E, G), whereas no differences were present in $\mathrm{CD} 4+\mathrm{CD} 69$ + or CD8 + CD69 + cells between septic $\mathrm{MLCK}^{-/-}$mice and WT mice (Figures 9 F, H).

\section{DISCUSSION}

This study demonstrates that sepsisinduced intestinal hyperpermeability is prevented in mice with genetic deletion 

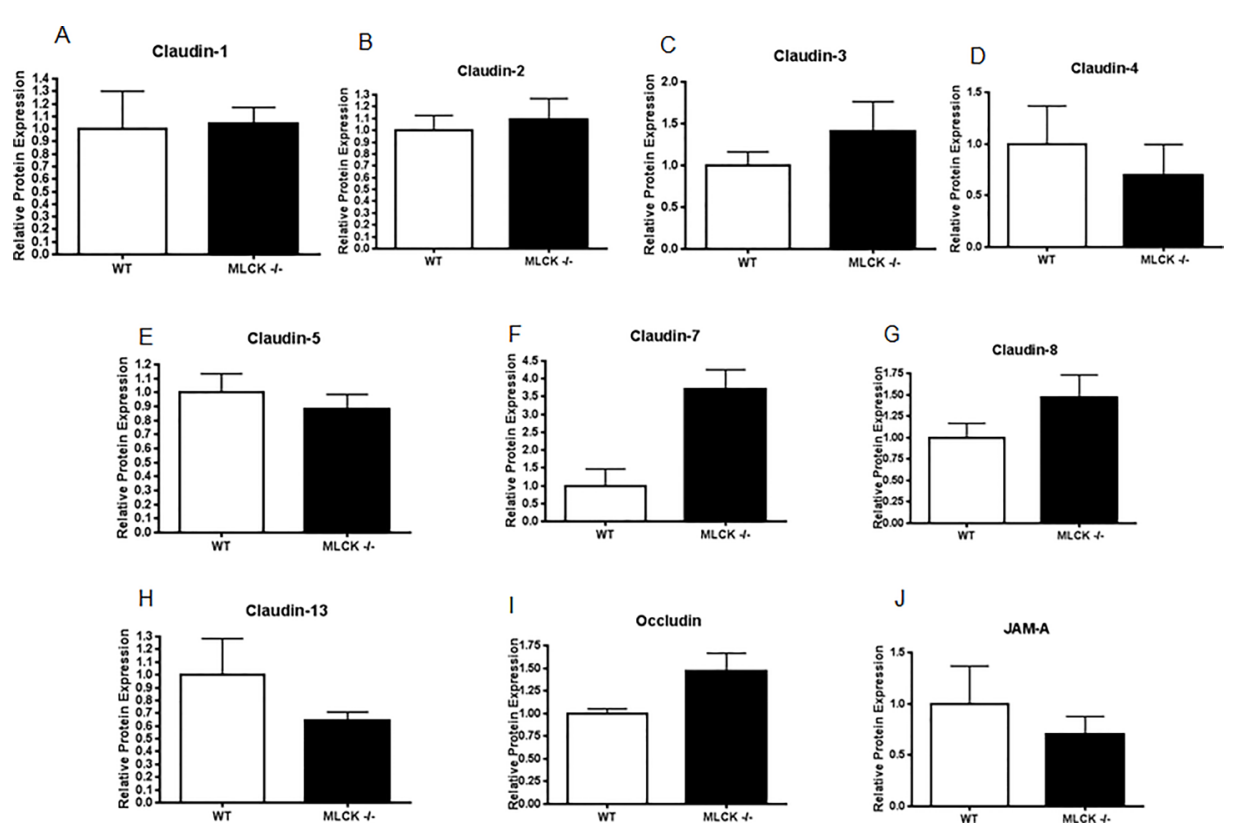

Figure 4. Effect of genetic deletion of MLCK on claudin 1, 2, 3, 4, 5, 7, 8 and 13, occludin and JAM-A 24 h after CLP. (A-J) No differences were seen in any of these tight junctions $(n=3-6 /$ group, $p=0.82,0.47,0.51,0.67,0.24,0.057,0.24,0.48,0.19,0.83$, respectively).
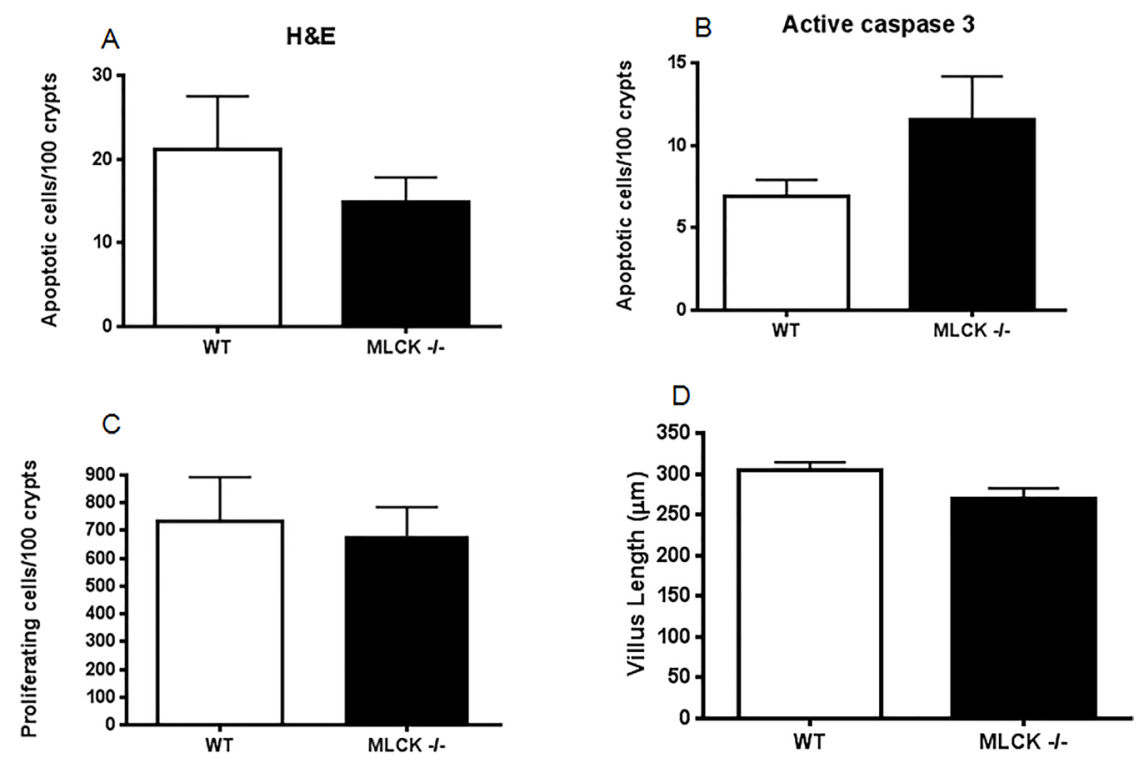

Figure 5. Effect of genetic deletion of MLCK on intestinal integrity $24 \mathrm{~h}$ after CLP. Intestinal apoptosis was similar between $\mathrm{MLCK}^{-1-}$ and WT mice following CLP whether measured by (A) H\&E ( $n=12$ /group, $p=0.58$ ) or (B) active caspase 3 ( $n=8-9 /$ group, $p=0.07$ ). (C) Proliferation as measured by number of S-phase cells $(n=8-9 /$ group, $p=0.37$ ) and (D) villus length ( $n=12 /$ group, $p=0.07$ ) were also similar between septic $\mathrm{MLCK}^{-1-}$ and WT mice.

of MLCK. This is associated with increases in ZO-1 and claudin 15. Notably, survival was markedly improved in $\mathrm{MLCK}^{-/-}$mice following CLP. The survival advantage was associated with a significant decrease in systemic IL-10 but was not accompanied by a decrease in either local or distant bacterial burden.
Intestinal barrier dysfunction in sepsis can occur through multiple mechanisms. The initial hyperinflammatory state in sepsis is associated with an increase in proinflammatory cytokines (49) that can activate MLCK (50). Invasive bacteria also induce redistribution of tight junction proteins and activation of MLCK (30). Finally, increased intestinal epithelial apoptosis can lead to increases in paracellular permeability $(51,52)$. Extensive literature indicates that sepsis is associated with a marked upregulation of inflammation, altered microbiome and gut epithelial apoptosis, and it is likely that all of these play a role in sepsis-induced intestinal hyperpermeability. However, our experiments yield some potential insights into which of these are most relevant in modulating permeability in WT and $\mathrm{MLCK}^{-/-}$mice. IL-6 induces barrier dysfunction in the setting of intestinal inflammation, and neutralization of IL-6 prevents intestinal hyperpermeability and MLCK expression in a mouse model of colitis (53). Our finding that IL-6 was lower in the blood and peritoneal fluid of $\mathrm{MLCK}^{-/-}$mice therefore represents a potential mechanism through which permeability could be reduced, by preventing the feed-forward worsening of barrier function induced by proinflammatory cytokines. In contrast, the finding that intestinal epithelial apoptosis was not different between septic WT and $\mathrm{MLCK}^{-/-}$mice makes it unlikely that normalization of intestinal barrier function in knockout mice was directly related to epithelial cell death. Further, quantitative cultures of peritoneal fluid and blood were similar in WT and $\mathrm{MLCK}^{-/-}$mice, suggesting that bacterial burden alone was not responsible for differences in barrier function, although we cannot rule out the possibility that the microbiome was more virulent in WT mice $(24,54)$, leading to more invasive bacteria - and hence hyperpermeability - in the absence of differences in bacterial numbers.

Hyperpermeability induced by CLP in WT mice is associated with increases in jejunal claudin 2 and JAM-A as well 

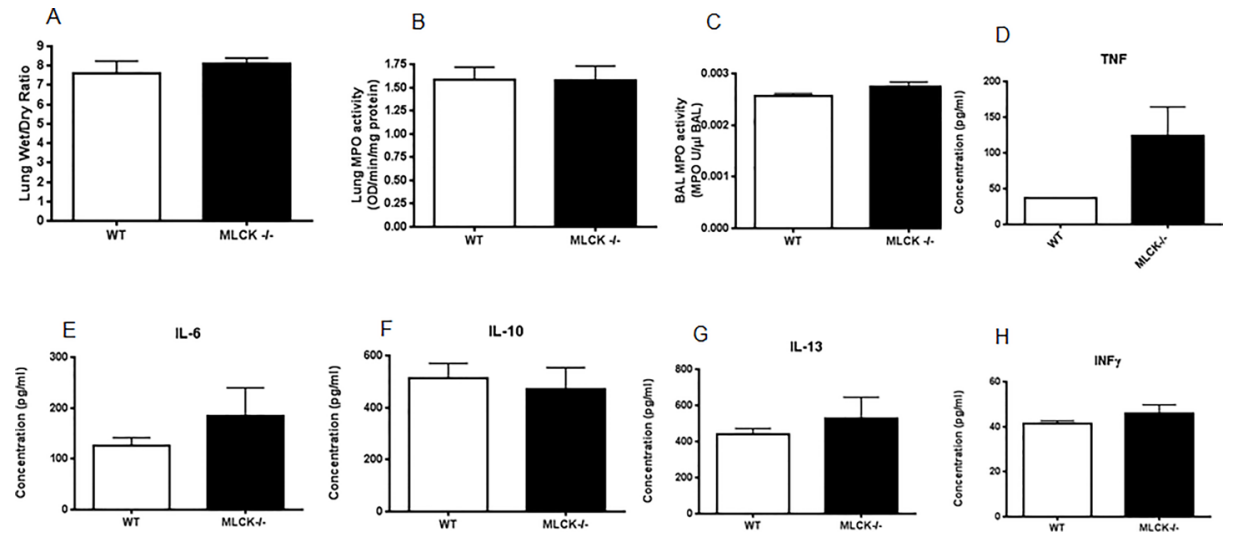

Figure 6. Effect of genetic deletion of MLCK on lung injury $24 \mathrm{~h}$ after CLP. (A) Lung wet-to-dry ratio was similar between $\mathrm{MLCK}^{-1-}$ and WT mice following CLP ( $n=10 /$ group, $p=0.78)$ as were (B) lung ( $n=8 /$ group, $p=0.85$ ) and $(C) B A L M P O(n=8 /$ group, $p=0.10)$. Concentrations in BAL fluid of (D) TNF ( $n=7$ /group, $p=0.46)$, (E) IL-6 ( $n=6 /$ group, $p=0.81$ ), (F) IL-10 ( $n=7-8 /$ group, $p=0.47)$, (G) IL-13 ( $n=7-8 /$ group, $p>0.99)$ and (H) IFN- $\gamma$ $\left(n=8 /\right.$ group, $p=0.37$ ) were all statistically similar between $\mathrm{MLCK}^{-1-}$ and WT mice.
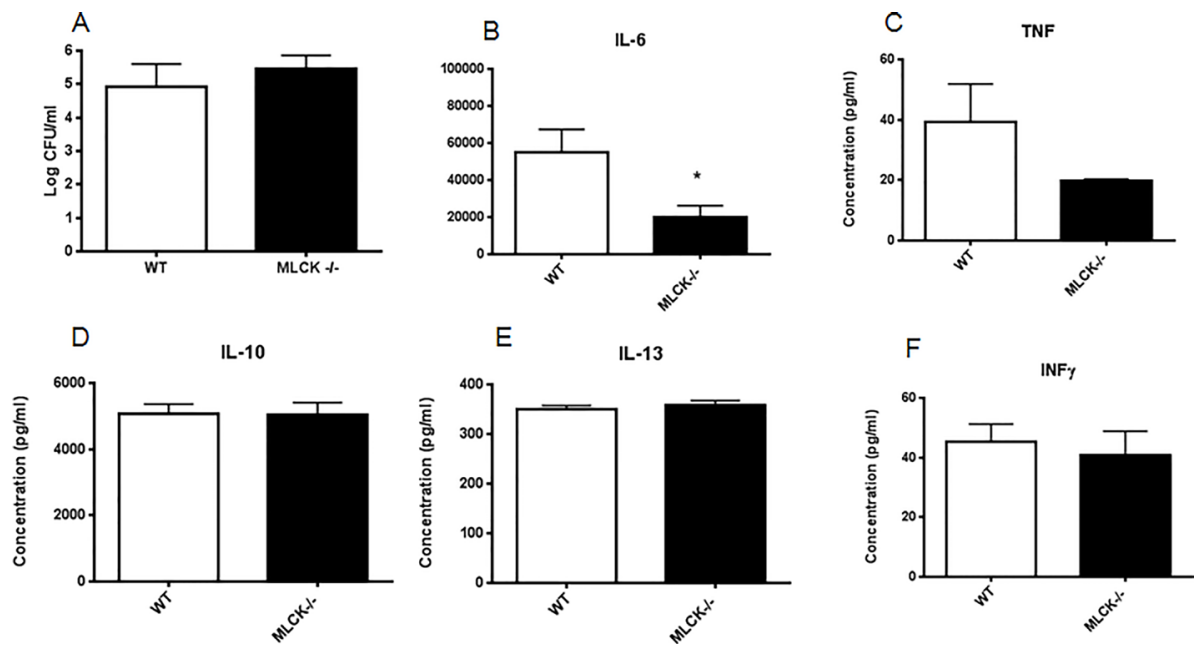

Figure 7. Effect of genetic deletion of MLCK on local bacterial burden and cytokines $24 \mathrm{~h}$ after CLP. (A) The number of bacteria in the peritoneal cavity was similar between $\mathrm{MLCK}^{-1-}$ and WT mice following CLP ( $n=10 /$ group, $\left.p=0.82\right)$. (B) IL-6 levels in peritoneal fluid were lower in $\mathrm{MLCK}^{-1-}$ mice $(n=6-9 /$ group, $p=0.03)$, but no differences were detected in (C) peritoneal TNF ( $n=8-9 /$ group, $p=0.17)$, (D) IL-10 $(n=10 /$ group, $p=0.76)$, (E) IL-13 ( $n=10 /$ group, $p=0.46)$ and $(F)$ IFN- $\gamma(n=10 /$ group, $p=0.65)$.

as decreases in claudin 5 and occludin (18). CLP has also been demonstrated to induce alterations in cellular localization of colonic claudins $1,3,4,5$ and 8 as well as upregulation of claudin 2 (21). Interestingly, none of these tight junction proteins was normalized in septic $\mathrm{MLCK}^{-/-}$mice, despite permeability in these animals being similar to unmanipulated WT and $\mathrm{MLCK}^{-/-}$mice. Instead, ZO-1 and claudin 15 were both increased in septic $\mathrm{MLCK}^{-/-}$mice. The increase in ZO-1 expression is consistent with previous studies of $\mathrm{MLCK}^{-/-}$mice (40) and alterations in this component of the tight junction likely represent a mechanism through which permeability is normalized in $\mathrm{MLCK}^{-/-}$mice.
Relatively little is known about claudin 15 in comparison. Claudin 15 (along with claudin 2) is unique in that it increases permeability to monovalent cations, and mice that are genetically deficient in claudin 15 have abnormally low sodium in the small intestine lumen and impaired glucose absorption (55). While the crystal structure for claudin 15 was recently identified (56), its relationship to MLCK is currently unclear, other than a single report demonstrating an association between increased MLCK and decreased claudin 15 in grass carp with niacin deficiency (57).

The survival advantage conferred by MCLK knockout was striking, with essentially all $\mathrm{MLCK}^{-/-}$mice surviving after CLP compared with only $24 \%$ of WT mice. While the simplest explanation for this would be that normalized permeability prevents bacterial translocation, our findings do not support this, since blood cultures demonstrated similar numbers of bacteria in both WT and $\mathrm{MLCK}^{-/-}$mice. This is consistent with studies in critically ill patients, suggesting that translocation of intact bacteria occurs infrequently (22). To look for potential causes of improved mortality, we analyzed the local and systemic inflammatory and immune response and lung injury. The rationale behind this experimental design was related to existing literature linking (1) intestinal permeability and apoptosis (discussed above), (2) MLCK and systemic inflammation $(53,58)$ and (3) lung injury from intestine-derived toxic factors via mesenteric lymph (59). The majority of the endpoints assayed were not different between WT and $\mathrm{MLCK}^{-/-}$mice, suggesting that they were not responsible for the differences in mortality. It is also important to note that permeability, gut apoptosis, proliferation, villus length, lung MPO and BAL MPO are similar between unmanipulated WT and $\mathrm{MLCK}^{-/-}$mice (Figure 2 and Supplementary Figure 1). In contrast, systemic IL-10 levels were greater than 10-fold lower in septic $\mathrm{MLCK}^{-/-}$mice compared with septic WT mice, even though baseline IL-10 

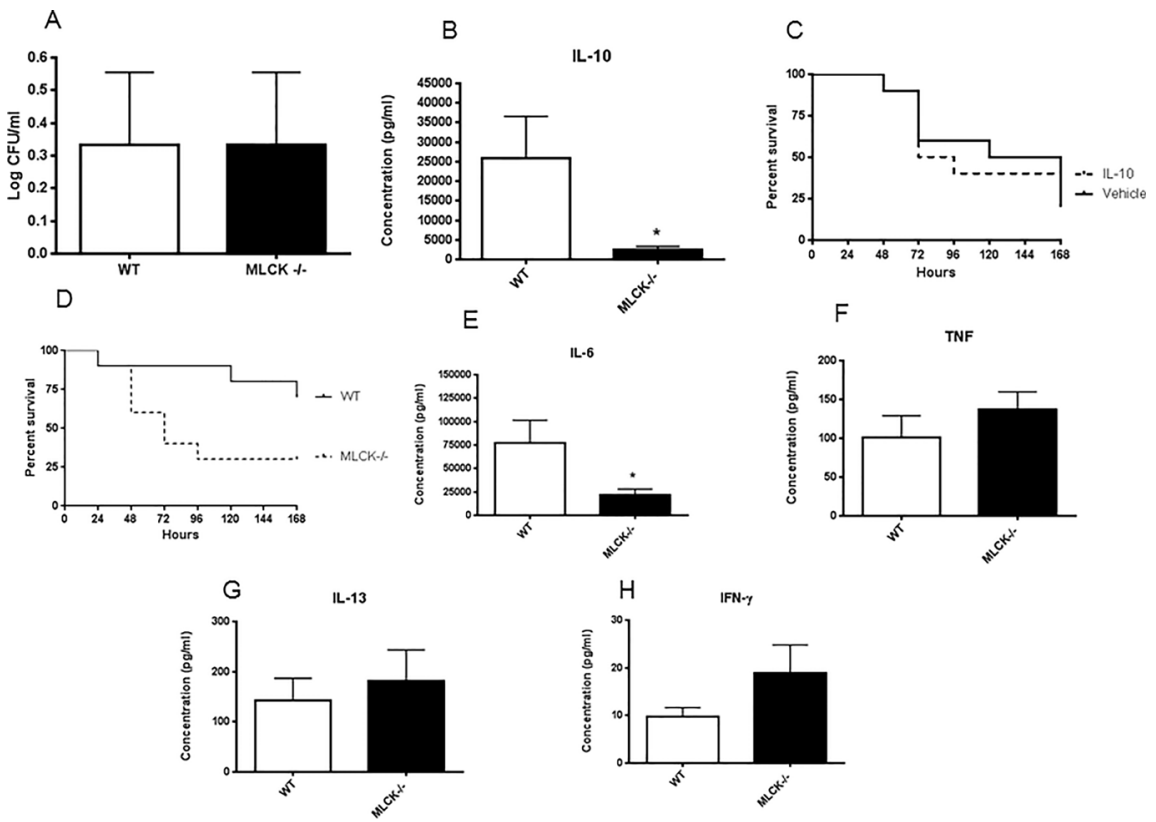

Figure 8. Effect of genetic deletion of MLCK on systemic bacterial burden and cytokines $24 \mathrm{~h}$ after CLP. (A) The amount of bacteria in the blood was similar between $\mathrm{MLCK}^{-1}$ and WT mice following CLP $(n=10 /$ group, $p>0.99)$. (B) IL-10 levels in the blood were significantly lower in $\mathrm{MLCK}^{-1-}$ mice ( $n=7-8 /$ group, $p=0.001$ ). (C) No differences in 7 d survival were identified when $\mathrm{MLCK}^{-1}$ mice were given exogenous IL-10 or vehicle ( $n=10 /$ group, $p=0.68)$. (D) The survival benefit conferred after CLP in $\mathrm{MLCK}^{-1-}$ disappeared when anti$\mathrm{IL}-10$ antibody was given to both $\mathrm{MLCK}^{-1}$ and WT mice $(n=10 /$ group, $p=0.05)$. (E) IL-6 levels were also lower in $\mathrm{MLCK}^{-1-}$ mice $(n=9-11 /$ group, $p=0.03)$. No differences were detected in systemic (F) TNF ( $n=8-9 /$ group, $p=0.14)$, (G) IL-13 ( $n=12 /$ group, $p=0.83$ ) and (H) IFN- $\gamma(n=12 /$ group, $p=0.08)$.
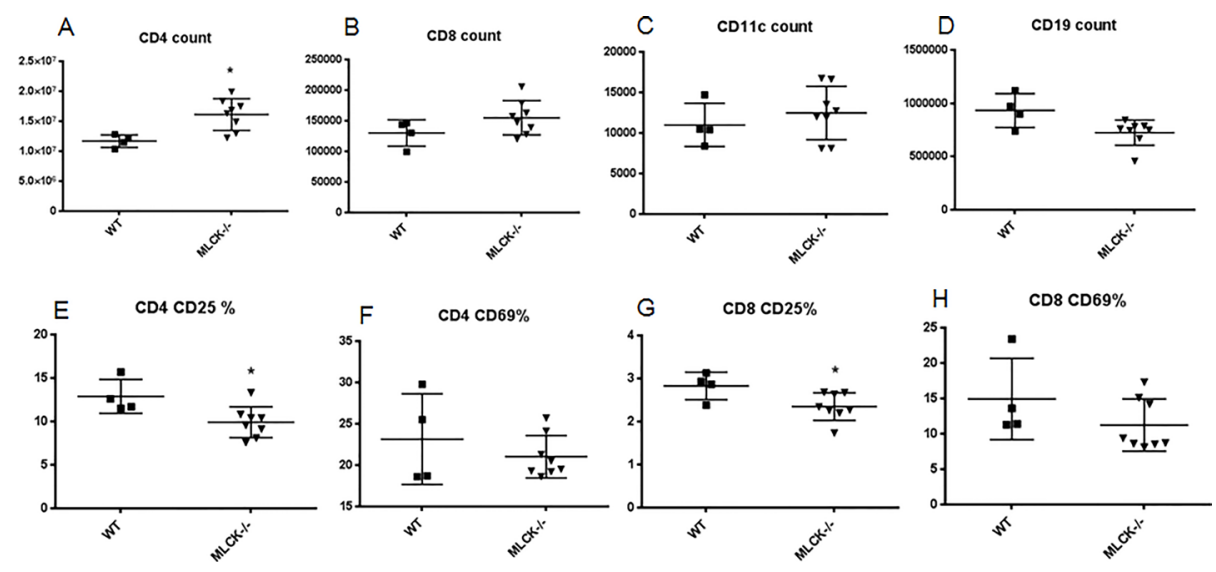

Figure 9. Effect of genetic deletion of MLCK on adaptive immune system $24 \mathrm{~h}$ after CLP. (A) CD4 + counts were higher in septic MLCK ${ }^{-1-}$ mice $(p=0.008)$, whereas $(B) C D 8+(p=0.20)$, (C) $C D 11 c+(n=4-8 /$ group, $p=0.51)$ and (D) $C D 19+(p=0.11)$ counts were similar between septic $\mathrm{MLCK}^{-1}$ and WT mice. (E) The percentage of CD4 + CD25 + cells was lower in septic $\mathrm{MLCK}^{-1-}$ mice $(p=0.02)$, while there was $(F)$ no difference in CD4 + CD69 + cells $(p=0.97)$. Similarly, $(G)$ the percentage of CD8 + CD25 + cells was lower in septic MLCK ${ }^{-1-}$ mice $(p=0.03)$ while there was $(H)$ no difference in CD8 + CD69 + cells $(p=0.28)$. levels were similar in unmanipulated $\mathrm{MLCK}^{-/-}$and WT mice. The relationship between IL-10 and sepsis is complex. Alterations in IL-10 single-nucleotide polymorphisms and systemic levels of IL-10 correlate with outcome in septic patients. Further, manipulating the cytokine via exogenous delivery, anti-IL10 antibody administration or genetic deletion improves, worsens or has no effect on survival in animal studies, depending on timing of administration and the sepsis model examined (60-65). To examine the role of IL-10 in mediating mortality in septic $\mathrm{MLCK}^{-/-}$mice, we performed experiments to both augment and neutralize IL-10. Since exogenous IL-10 did not worsen outcome in septic $\mathrm{MLCK}^{-/-}$mice compared with vehicle, we cannot conclude that the decreased levels of IL-10 seen in knockout mice were responsible for the improved outcome, since, in theory, if IL-10 were maladaptive, decreasing levels would be beneficial while increasing levels would be harmful. In addition, the survival benefit conferred in $\mathrm{MLCK}^{-/-}$mice was completely eliminated when IL-10-neutralizing antibody was given to both WT and $\mathrm{MLCK}^{-/-}$mice (and, in fact, there was a trend toward worsened survival in $\mathrm{MLCK}^{-/-}$mice), suggesting that it is plausible that IL-10 plays a role in the differential mortality observed. However, it is notable that IL-10 levels were already low in $\mathrm{MLCK}^{-/-}$mice, and the biological significance of neutralizing an already decreased level of IL-10 is not clear. Of note, our results are generally consistent with a previous study by Ranaivo et al. examining $\mathrm{MLCK}^{-/-}$mice subjected to a more lethal model of CLP, which demonstrated a modest improvement in survival without examining any endpoints in CLP beyond mortality (66). However, that study showed a smaller (although still significant) improvement in survival, from approximately $10 \%$ to $50 \%$. There are multiple potential reasons for this discrepancy. First, the current study used antibiotics, whereas the Ranaivo study did not, and antibiotics significantly alter outcomes from CLP. Next, the needle 
gauge was different in each experiment (25 versus 23 ), which could have altered the amount of intraperitoneal contamination. Considering that source control is a significant determinant of mortality following CLP (67), it is possible that a higher degree of soilage in the Ranaivo et al. study made it more difficult to rescue animals. Further, the mice used in this study were from the same animal facility and room but were not littermates, whereas the previous study used actual littermates, and differences in microbiota might have accounted for the differential mortality.

While the majority of immune parameters examined were not different between $\mathrm{MLCK}^{-/-}$and WT mice, a few differences were noted. First, numbers of CD4 + T cells were higher in $\mathrm{MLCK}^{-/-}$mice. Even though sepsis induces CD4 + T cell apoptosis, which could theoretically account for the observed difference between knockout and WT mice, it is important to note that $\mathrm{MLCK}^{-/-}$mice have higher CD4 + T cell counts at baseline compared with WT mice (data not shown; [68]), so it is not clear whether sepsis plays a role in the augmentation of CD4 + T cells. There was also a small but statistically significant decrease in the percentage of CD4 + CD25 + T cells in $\mathrm{MLCK}^{-/-}$mice. This difference is not present at baseline (data not shown), so it is possible that $\mathrm{MLCK}^{-/-}$mice have a less suppressive phenotype in the setting of sepsis. However, further immunophenotyping and functional analysis of the host response in septic $\mathrm{MLCK}^{-/-}$mice is required to understand the significance of these findings.

This study has a number of limitations. The most obvious is that the $\mathrm{MLCK}^{-/-}$ mice have genetic deletion of MLCK in all cell types. Since MLCK has been shown to be altered in vascular smooth muscle in septic patients and alveolar epithelial monolayers derived from septic rats $(69,70)$, it is possible that the survival benefit conferred in $\mathrm{MLCK}^{-/-}$mice is mechanistically unrelated to the endpoints examined. Further, since $\mathrm{MLCK}^{-/-}$ mice have a genetic deletion prior to the onset of sepsis, it is possible that this preexisting deficiency created an environment in which sepsis could not progress, a scenario with questionable clinical significance. Although we dosed exogenous IL-10 based upon a published study in Acinetobacter baumannii-induced sepsis (48), we cannot conclude definitively that this was the correct dosing strategy to test the role of IL-10 in mediating the mortality benefit conferred by knocking out MLCK. In addition, all animals were bred to mice of the same genotype. Thus, although $\mathrm{MLCK}^{-/-}$and WT mice were both kept in the same animal facility in the same room throughout the course of all experiments, they were not littermates and it is possible that differences in their microbiota could have played a role in the survival differences seen. Next, baseline mortality for septic $\mathrm{MLCK}^{-/-}$mice was different in Figure 1 and Figure 8, so the survival data must be interpreted with caution. It is possible this is the result of different surgical technique (CAL performed the survival curve in Figure 1; ZL performed the survival curve in Figure 8C) or some degree of inherent variation in the CLP model. Finally, animals were assayed for mechanistic endpoints at only a single time point $(24 \mathrm{~h})$ in an effort to minimize animal usage, and it is likely that we missed important information by not conducting a time series experiment.

\section{CONCLUSION}

These studies demonstrate that knocking out MLCK is associated with a marked improvement in survival, which is associated with normalization of intestinal permeability. Improving intestinal permeability represents an attractive strategy for the treatment of sepsis, and MLCK is a potential specific target, although further studies are required to determine the generalizability of our results.

\section{ACKNOWLEDGMENTS}

This work was supported by funding from the National Institutes of Health (GM072808, GM095442, GM104323, GM109779, GM113228, HL116958).

\section{DISCLOSURE}

The authors declare they have no competing interests as defined by Molecular Medicine or other interests that may be perceived to influence the results and discussion reported in this paper.

\section{REFERENCES}

1. Singer M, et al. (2016) The third international consensus definitions for sepsis and septic shock (Sepsis-3). JAMA. 315:801-10.

2. Shankar-Hari M, et al. (2016) Developing a new definition and assessing new clinical criteria for septic shock: for the third international consensus definitions for sepsis and septic shock (Sepsis-3). JAMA. 315:775-87.

3. Gaieski DF, Edwards JM, Kallan MJ, Carr BG. (2013) Benchmarking the incidence and mortality of severe sepsis in the United States. Crit. Care Med. 41:1167-74.

4. Rhodes A, et al. (2017) Surviving sepsis campaign: international guidelines for management of sepsis and septic shock: 2016. Crit. Care Med. 45:486-552.

5. Mittal R, Coopersmith CM. (2014) Redefining the gut as the motor of critical illness. Trends Mol. Med. 20:214-23.

6. Klingensmith NJ, Coopersmith CM. (2016) The gut as the motor of multiple organ dysfunction in critical illness. Crit Care Clin. 32:203-12.

7. Carrico CJ, Meakins JL, Marshall JC, Fry D, Maier RV. (1986) Multiple-organ-failure syndrome. The gastrointestinal tract: the "motor" of MOF. Arch. Surg. 121:196-208.

8. Sertaridou E, Papaioannou V, Kolios G, Pneumatikos I. (2015) Gut failure in critical care: old school versus new school. Ann. Gastroenterol. 28:309-22.

9. Clark JA, Coopersmith CM. (2007) Intestinal crosstalk: a new paradigm for understanding the gut as the "motor" of critical illness. Shock. 28:384-93.

10. Fay KT, Ford ML, Coopersmith CM. (2017) The intestinal microenvironment in sepsis. Biochim. Biophys. Acta.

11. Lyons JD, Coopersmith CM. (2017) Pathophysiology of the Gut and the Microbiome in the Host Response. Pediatr. Crit. Care Med. 18:S46-49.

12. Clark JA, Clark AT, Hotchkiss RS, Buchman TG, Coopersmith CM. (2008) Epidermal growth factor treatment decreases mortality and is associated with improved gut integrity in sepsis. Shock. 30:36-42.

13. Clark JA, Gan H, Samocha AJ, Fox AC, Buchman TG, Coopersmith CM. (2009) Enterocyte-specific epidermal growth factor prevents barrier dysfunction and improves mortality in murine peritonitis. Am. J. Physiol Gastrointest. Liver Physiol. 297: G471-79.

14. Coopersmith CM, et al. (2002) Overexpression of $\mathrm{Bcl}-2$ in the intestinal epithelium improves survival in septic mice. Crit. Care Med. 30:195-201. 
15. Coopersmith CM, et al. (2003) Sepsis from Pseudomonas aeruginosa pneumonia decreases intestinal proliferation and induces gut epithelial cell cycle arrest. Crit. Care Med. 31:1630-37.

16. Dominguez JA, et al. (2013) Inhibition of IKKbeta in enterocytes exacerbates sepsis-induced intestinal injury and worsens mortality. Crit. Care Med. 41: e275-85.

17. Liang Z, et al. (2014) Intestine-specific deletion of microsomal triglyceride transfer protein increases mortality in aged mice. PLOS. ONE. 9:e101828.

18. Yoseph BP, et al. (2016) Mechanisms of intestinal barrier dysfunction in sepsis. Shock. In press.

19. Fink MP. (2003) Intestinal epithelial hyperpermeability: update on the pathogenesis of gut mucosal barrier dysfunction in critical illness. Curr. Opin. Crit Care 9:143-51.

20. Klaus DA, et al. (2013) Increased plasma zonulin in patients with sepsis. Biochem. Med. (Zagreb. ) 23:107-11.

21. Li Q, Zhang Q, Wang C, Liu X, Li N, Li J. (2009) Disruption of tight junctions during polymicrobial sepsis in vivo. J. Pathol. 218:210-21.

22. Moore FA, et al. (1991) Gut bacterial translocation via the portal vein: a clinical perspective with major torso trauma. J. Trauma 31:629-36.

23. Alverdy JC, Laughlin RS, Wu L. (2003) Influence of the critically ill state on host-pathogen interactions within the intestine: gut-derived sepsis redefined. Crit. Care Med. 31:598-607.

24. Krezalek MA, Defazio J, Zaborina O, Zaborin A, Alverdy JC. (2016) The shift of an intestinal "microbiome" to a "pathobiome" governs the course and outcome of sepsis gollowing surgical injury. Shock. 45:475-82.

25. Odenwald MA, Turner JR. (2013) Intestinal permeability defects: is it time to treat? Clin. Gastroenterol. Hepatol. 11:1075-83.

26. Nalle SC. (2014) Recipient NK cell inactivation and intestinal barrier loss are required for MHC-matched graft-versus-host disease. Sci. Transl. Med. 6:243ra87.

27. Turner JR. (2009) Intestinal mucosal barrier function in health and disease. Nat. Rev. Immunol. 9:799-809.

28. Dominguez JA, et al. (2011) Epidermal growth factor improves survival and prevents intestinal injury in a murine model of pseudomonas aeruginosa pneumonia. Shock. 36:381-89.

29. Chen C, Wang P, Su Q, Wang S, Wang F. (2012) Myosin light chain kinase mediates intestinal barrier disruption following burn injury. PLoS ONE. 7:e34946.

30. Barreau F, Hugot JP. (2014) Intestinal barrier dysfunction triggered by invasive bacteria. Curr. Opin. Microbiol. 17:91-98.

31. Herrmann JR, Turner JR. (2016) Beyond Ussing's chambers: contemporary thoughts on integration of transepithelial transport. Am. J. Physiol. Cell Physiol 310: C423-31.

32. Khounlotham M, et al. (2012) Compromised intestinal epithelial barrier induces adaptive immune compensation that protects from colitis. Immunity. 37:563-73.
33. Laukoetter MG, et al. (2007) JAM-A regulates permeability and inflammation in the intestine in vivo. J. Exp. Med. 204:3067-76.

34. Shen L, Weber CR, Raleigh DR, Yu D, Turner JR. (2011) Tight junction pore and leak pathways: a dynamic duo. Annu. Rev. Physiol 73:283-309.

35. Cunningham KE, Turner JR. (2012) Myosin light chain kinase: pulling the strings of epithelial tight junction function. Ann. N.Y. Acad. Sci. 1258:34-42.

36. Jung C, et al. (2012) Yersinia pseudotuberculosis disrupts intestinal barrier integrity through hematopoietic TLR-2 signaling. J. Clin. Invest 122:2239-51.

37. Meinzer U, et al. (2012) Yersinia pseudotuberculosis effector YopJ subverts the Nod2/RICK/TAK1 pathway and activates caspase- 1 to induce intestinal barrier dysfunction. Cell Host Microbe. 11:337-51.

38. Zahs A, et al. (2012) Inhibition of long myosin light-chain kinase activation alleviates intestinal damage after binge ethanol exposure and burn injury. Am. J. Physiol. Gastrointest. Liver Physiol. 303:G705-12.

39. Shen L. (2012) Tight junctions on the move: molecular mechanisms for epithelial barrier regulation. Ann. N.Y. Acad. Sci. 1258:9-18.

40. Yu D, et al. (2010) MLCK-dependent exchange and actin binding region-dependent anchoring of ZO-1 regulate tight junction barrier function. Proc. Natl. Acad. Sci. U.S.A. 107:8237-41.

41. Zolotarevsky Y, et al. (2002) A membrane-permeant peptide that inhibits MLC kinase restores barrier function in in vitro models of intestinal disease. Gastroenterology. 123:163-72.

42. Yuhan R, Koutsouris A, Savkovic SD, Hecht G. (1997) Enteropathogenic Escherichia coli-induced myosin light chain phosphorylation alters intestinal epithelial permeability. Gastroenterology. 113:1873-82.

43. Clayburgh DR, et al. (2005) Epithelial myosin light chain kinase-dependent barrier dysfunction mediates $\mathrm{T}$ cell activation-induced diarrhea in vivo. J. Clin. Invest. 115:2702-15.

44. Rittirsch D, Huber-Lang MS, Flierl MA, Ward PA. (2009) Immunodesign of experimental sepsis by cecal ligation and puncture. Nat. Protoc. 4:31-36.

45. Dellinger RP, et al. (2013) Surviving sepsis campaign: international guidelines for management of severe sepsis and septic shock: 2012. Crit. Care Med. 41:580-637.

46. Vyas D, et al. (2007) Epithelial apoptosis in mechanistically distinct methods of injury in the murine small intestine. Histol. Histopathol. 22:623-30.

47. Lyons JD, et al. (2016) Murine lung cancer increases CD4+ T cell apoptosis and decreases gut proliferative capacity in sepsis. PLOS ONE. 11:e0149069.

48. Noto MJ, Becker KW, Boyd KL, Schmidt AM, Skaar EP. (2017) RAGE-mediated suppression of interleukin-10 results in enhanced mortality in a murine model of cinetobacter baumannii sepsis. Infect. Immun. 85:e00954-16.
49. Hotchkiss RS, Sherwood ER. (2015) Immunology. Getting sepsis therapy right. Science. 347:1201-02.

50. Bruewer M, et al. (2003) Proinflammatory cytokines disrupt epithelial barrier function by apoptosis-independent mechanisms. J. Immunol. 171:6164-72

51. Abreu MT, Palladino AA, Arnold ET, Kwon RS, McRoberts JA. (2000) Modulation of barrier function during Fas-mediated apoptosis in human intestinal epithelial cells. Gastroenterology. 119:1524-36.

52. Chin AC, Flynn AN, Fedwick JP, Buret AG. (2006) The role of caspase- 3 in lipopolysaccharidemediated disruption of intestinal epithelial tight junctions. Can. J. Physiol. Pharmacol. 84:1043-50.

53. Xiao YT, Yan WH, Cao Y, Yan JK, Cai W. (2016) Neutralization of IL-6 and TNF-alpha ameliorates intestinal permeability in DSS-induced colitis. Cytokine. 83:189-92.

54. McDonald D, et al. (2016) Extreme dysbiosis of the microbiome in critical illness. mSphere. 1(4):e00199-16

55. Tamura A, et al. (2011) Loss of claudin-15, but not claudin-2, causes $\mathrm{Na}+$ deficiency and glucose malabsorption in mouse small intestine. Gastroenterology. 140:913-23.

56. Suzuki H, et al. (2014) Crystal structure of a claudin provides insight into the architecture of tight junctions. Science. 344:304-07.

57. Feng L, et al. (2016) Deficiency of dietary niacin impaired intestinal mucosal immune function via regulating intestinal NF-kappaB, Nrf2 and MLCK signaling pathways in young grass carp (Ctenopharyngodon idella). Fish Shellfish Immunol. 49:177-93.

58. Du J, et al. (2015) 1,25-Dihydroxy vitamin D protects intestinal epithelial barrier by regulating the myosin light chain kinase signaling pathway. Inflamm. Bowel. Dis. 21:2495-06.

59. Deitch EA. (2010) Gut lymph and lymphatics: a source of factors leading to organ injury and dysfunction. Ann. N.Y. Acad. Sci. 1207(Suppl 1):E103-11.

60. Remick DG, et al. (1998) Exogenous interleukin-10 fails to decrease the mortality or morbidity of sepsis. Crit. Care Med. 26:895-904.

61. Montoya-Ruiz C, et al. (2016) Variants in LTA, TNF, IL1B and IL10 genes associated with the clinical course of sepsis. Immunol. Res. 64:1168-78.

62. Rajan S, et al. (2007) Intestine-specific overexpression of IL-10 improves survival in polymicrobial sepsis. Shock. 45:486-522.

63. Deng M, et al. (2016) Toll-like receptor 4 signaling on dendritic cells suppresses polymorphonuclear leukocyte CXCR2 expression and trafficking via interleukin 10 during intra-abdominal sepsis. J. Infect. Dis. 213:1280-88.

64. van der Poll T, et al. (1995) Endogenous IL-10 protects mice from death during septic peritonitis. J. Immunol. 155:5397-5401.

65. Murphey ED, Sherwood ER. (2006) Bacterial clearance and mortality are not improved by a combination of IL-10 neutralization and IFNgamma administration in a murine model of post-CLP immunosuppression. Shock. 26:417-24. 
66. Ralay RH, et al. (2007) Protection against endotoxic shock as a consequence of reduced nitrosative stress in MLCK210-null mice. Am. J. Pathol. 170:439-46.

67. Alverdy JC, Krezalek MA. (2017) Collapse of the microbiome, emergence of the pathobiome, and the immunopathology of sepsis. Crit. Care Med. 45:337-47.

68. Su L, et al. (2009) Targeted epithelial tight junction dysfunction causes immune activation and contributes to development of experimental colitis. Gastroenterology. 136:551-63.

69. Zheng W, Kou Y, Gao FL, Ouyang XH. (2016) Enzymatic changes in myosin regulatory proteins may explain vasoplegia in terminally ill patients with sepsis. Biosci. Rep. 36(2):e00305.

70. Cohen TS, DiPaolo BC, Lawrence GG,

Margulies SS. 2012. Sepsis enhances epithelial permeability with stretch in an actin dependent manner. PLOS ONE. 7:e38748.

Cite this article as: Lorentz CA, et al. (2017) Myosin light chain kinase knockout improves gut barrier function and confers a survival advantage in polymicrobial sepsis. Mol. Med. 23:155-65. 